\title{
Systemic Inflammation and Reperfusion Injury in Patients With Acute Myocardial Infarction
}

\author{
Fien Blancke, ${ }^{1}$ Marc J. Claeys, ${ }^{1}$ Philippe Jorens, ${ }^{2}$ Guy Vermeiren, ${ }^{1}$ Johan Bosmans, ${ }^{1}$ Floris L. Wuyts, ${ }^{3}$ and Chris J. Vrints ${ }^{1}$ \\ ${ }^{1}$ Department of Cardiology, Antwerp University Hospital, University of Antwerp (UA), \\ Wilrijkstraat 10, 2650 Edegem, Belgium \\ ${ }^{2}$ Department of Intensive Care Medicine, Antwerp University Hospital, University of Antwerp (UA), \\ Wilrijkstraat 10, 2650 Edegem, Belgium \\ ${ }^{3}$ Department of Biomedical Physics and Biostatistics, Antwerp University Hospital, University of Antwerp (UA), \\ Wilrijkstraat 10, 2650 Edegem, Belgium
}

Received 15 July 2005; accepted 18 August 2005

\begin{abstract}
Despite early recanalization of an occluded infarct artery, tissue reperfusion remains impaired in more than one-third of the acute myocardial infarction (AMI) patients owing to a process of reperfusion injury. The role of systemic inflammation in triggering this phenomenon is unknown. Proinflammatory factors (hs-CRP, TNF- $\alpha$ ) and anti-inflammatory mediators (IL-1 receptor antagonist, IL-10) were measured in 65 patients during the acute phase of a myocardial infarction as well as in 11 healthy control subjects. Myocardial reperfusion injury was defined as the presence of persistent ST-segment elevation despite successful coronary intervention ( $\geq 50 \%$ of the initial value) and was observed in 28 patients. Systemic proinflammatory mediators (particularly hs-CRP and leukocytes) were higher in AMI patients compared to control subjects. Within the group of AMI patients, only serum TNF- $\alpha$ differed significantly between patients with versus without reperfusion injury: a median value of 25 versus $13 \mathrm{pg} / \mathrm{mL}$ was observed, respectively. Logistic regression analysis identified a high level of TNF- $\alpha$ as the most important independent determinant of reperfusion injury $(P=.001)$, beyond total ischemic time $(P=.01)$ and extent of jeopardized myocardium $(P=.08)$. There was no correlation between the TNF- $\alpha$ level and the total ischemic time $(P=.8)$ or the extent of jeopardized myocardium $(P=.6)$. Systemic inflammation, in particular high levels of TNF- $\alpha$, is strongly associated with the occurrence of reperfusion injury after successful recanalization. Our findings suggest that TNF- $\alpha$ is involved in the triggering and/or amplification of local inflammatory responses related to ischemia-reperfusion injury.
\end{abstract}

\section{INTRODUCTION}

Despite successful early recanalization of an occluded infarct artery by percutaneous coronary intervention (PCI) or thrombolysis, up to one-third of AMI patients still fail to obtain complete myocardial reperfusion due to a process of myocardial reperfusion injury $[1,2]$. This phenomenon is characterised by an impairment of microcirculatory flow and by ongoing ischemia and tissue necrosis $[3,4]$. Although the underlying mechanisms of reperfusion injury are still not fully elucidated, there is accumulating evidence that local inflammatory responses with infiltration of leucocytes in the capillary circulation and release of oxygen-free radicals play a key role in this reperfusion-related tissue injury $[5,6]$. However, the occurrence and the extent of reperfusion injury is variable and is not solely related to the severity and duration of myocardial ischemia $[2,7]$. Whether the pre-existing sys-

Correspondence and reprint requests to Marc J. Claeys, Department of Cardiology, Antwerp University Hospital, University of Antwerp (UA), Wilrijkstraat 10, 2650 Edegem, Belgium; marc.claeys@ua.ac.be temic inflammatory state might be another important determinant of this phenomenon is unknown.

Accordingly, this study was designed to compare the systemic inflammatory state in AMI patients with versus without reperfusion injury. Systemic inflammatory state was evaluated in the acute phase by measuring both proinflammatory mediators (hs-CRP, TNF- $\alpha$ ) as antiinflammatory mediators (IL-1 receptor antagonist, IL10).

\section{METHODS \\ Patient population}

Between September 2003 and April 2004, a total of 65 patients with AMI lasting < 12 hours were prospectively and consecutively included and underwent immediate coronary angiography and percutaneous coronary intervention if needed. All included patients showed initial $>0.1 \mathrm{mV}$ ST-segment elevation in at least two contiguous electrocardiographic leads and had 12-lead electrocardiogram available before and at the end of the coronary intervention. All patients showed adequate restoration of coronary patency (thrombolysis in myocardial infarction 
(TIMI) flow grade $\geq 2$ ) at the end of the procedure. Patients with left bundle-branch block or with poorly interpretable ST-segments, were excluded. Arterial blood samples for measurements of the inflammatory status were collected at the time of cardiac catheterisation and were stored at $-80^{\circ} \mathrm{C}$ after centrifugation. The study group was stratified according to the presence or absence of reperfusion injury as was assessed on serial ST-segment measurements. The data of this study group were compared with a control group of 11 healthy subjects.

The study was approved by the institutional ethical committee of the Antwerp University Hospital and informed consent was obtained from all patients.

\section{Electrocardiographic analysis}

To assess the presence of myocardial reperfusion injury, serial ST-segment analysis on a 12-lead electrocardiogram was obtained before and after successful recanalisation of the infarct artery (i.e., before discharge from the catheterisation laboratory). The sum of the ST-segment elevations was measured manually with lens-intensified calipers 20 milliseconds after the end of the QRS complex from leads I, aVL, and V1 through V6 for left anterior descending artery occlusions and leads II, III, aVF, V5, V6, and reciprocal ST-segment depressions in V1-2 for right coronary/left circumflex occlusions. Persistent STsegment elevation, expressed as a percentage of the initial ST-segment elevation (\%ST), was used as an electrical marker of reperfusion injury and has been shown to correlate well with impaired myocardial reperfusion at the microcirculatory level $[7,8]$. Persistent ST-segment elevation, equal or more than $50 \%$ of the initial value (ST = $50 \%$ ) was defined as severely impaired myocardial reperfusion with ongoing tissue injury. On the other hand, STsegment elevations of less than $50 \%$ (ST $<50 \%)$ indicated good myocardial reperfusion without significant ongoing tissue injury. We have demonstrated a good reproducibility of these measurements in previous reports [9].

\section{Laboratory data}

Measurements of tumor necrosis factor alfa (TNF- $\alpha$ ), interleukin- 10 (IL-10), interleukin-1 receptor antagonist (IL-1ra) were performed using ELISA with reagents from Bender MedSystems (Vienna, Austria) (for TNF- $\alpha$ and IL10) and R \& D Systems (Minneapolis, Minn, USA) (for IL-1 ra). The intra-assay variances for all ELISA measurements were $<5 \%$. Plasma CRP was measured by a highsensitivity latex particle turbidimetric assay (Wakao). The lower limit of sensitivity of this method is $0.01 \mathrm{mg} / \mathrm{dL}$.

\section{Angiographic data}

Coronary angiographic data were quantitatively analysed with a computer-based cardiovascular angiography analysis system (CAAS II, Pie Medical Data, The Netherlands). Multivessel disease was defined as the presence of a lesion with $>50 \%$ diameter stenosis in a noninfarct related coronary artery. The thrombolysis in myocardial infarction (TIMI) angiographic scale was used to determine the recanalisation status of the infarct-related artery and was assessed visually. TIMI blush grade was applied to assess microcirculatory flow after intervention [10]. TIMI blush grade 0 - 1 reflects poor myocardial perfusion.

\section{Clinical data}

The total ischemic time could be registered adequately in 64 patients and was defined as the time from the beginning of pain to the recanalisation of the occluded infarct artery. In case of an open artery at the time of cardiac catheterization, total ischemic time was defined as the time from the beginning of the pain until resolution of pain after initiation of thrombolytic therapy.

Treatment with thrombolytic therapy, glycoprotein IIb/IIIa receptor antagonists, and stent use were left at the discretion of the treating physician.

Patients were followed up to one month with data being recorded from clinic visits and/or telephone calls to the referral physician. Two major adverse cardiac events (MACE) were identified: cardiac death including sudden death without evidence of a noncardiac origin and nonfatal myocardial infarction. Myocardial infarction was defined by enzymatic or electrocardiographic documentation during hospital admission. Only one event, the most serious in the above order, was tabulated for each patient.

\section{Statistical analysis}

Continuous variables are presented as median value with 25th and 75th percentiles and comparisons between groups were made with the Mann-Whitney $U$ test. Differences between proportions were assessed by chi-square analysis. Spearman's rank correlation method was used to correlate TNF- $\alpha$ levels with ischemic time and extent of jeopardized myocardium (expressed as summation of STsegment elevation before intervention).

To identify the independent determinants of myocardial reperfusion injury, logistic regression analysis was applied including inflammatory parameters, age, presence of diabetes, total ischemic time, Killip class, extent of jeopardized myocardium, use of adjunctive therapy with glycoprotein IIb/IIIa inhibitors and stents, diameter stenosis post PCI, and extent of coronary artery disease. A $P$ value $<.05$ was considered statistically significant.

\section{RESULTS}

\section{Characteristics of study population}

A total of 37 AMI patients showed ECG signs of good reperfusion whereas 28 AMI patients had ECG signs of reperfusion injury. Table 1 summarizes the baseline characteristics of these two study groups. Patients with reperfusion injury, as compared to patients with good reperfusion, were older and were admitted more frequently with cardiac failure or cardiogenic shock (cf Killip class $>1$ ). Total ischemic time was longer in patients with reperfusion injury and more patients with reperfusion injury 
TABLE 1. Baseline characteristic. Data are presented as median value with 25 th and 75 th percentiles.

\begin{tabular}{|c|c|c|c|}
\hline Variable & Impaired reperfusion $(n=28)$ & Good reperfusion $(n=37)$ & $P$ value \\
\hline Age $(y)$ & $69(53,75)$ & $60(55,66)$ & .17 \\
\hline Men & $24(86 \%)$ & $29(78 \%)$ & .4 \\
\hline Hypertension & $16(57 \%)$ & $20(54 \%)$ & .8 \\
\hline Cholesterolemia > $220 \mathrm{mg} \%$ & $15(45 \%)$ & $16(44 \%)$ & .5 \\
\hline Lipid-lowering therapy & $4(15 \%)$ & $7(19 \%)$ & .7 \\
\hline Diabetes mellitus & $4(14 \%)$ & $2(5 \%)$ & .2 \\
\hline Current smoker & $11(39 \%)$ & $14(38 \%)$ & .9 \\
\hline Killip class > 1 & $5(18 \%)$ & $0(0 \%)$ & .01 \\
\hline Total ischemic time (s) & $270(180,382)$ & $180(150,285)$ & .01 \\
\hline Thrombolysis & $14(50 \%)$ & $17(47 \%)$ & .8 \\
\hline Systolic pressure (mmHg) & $130(110,141)$ & $120(106,137)$ & .6 \\
\hline GP IIb/IIIa inhibitor & $13(48 \%)$ & $25(67 \%)$ & .1 \\
\hline Multivessel coronary disease & $11(39 \%)$ & $17(46 \%)$ & .6 \\
\hline Infarct coronary artery & - & - & - \\
\hline RCA/LAD/CX (\%) & $46 / 46 / 8$ & $33 / 40 / 27$ & .1 \\
\hline TIMI flow pre-PCI, $0 / 1 / 2 / 3(\%)$ & $54 / 7 / 21 / 18$ & $46 / 13 / 21 / 18$ & .8 \\
\hline TIMI flow post-PCI 2/3 (\%) & $44 / 56$ & $14 / 86$ & .005 \\
\hline MBG post-PCI, 0/1/2/3 (\%) & $7 / 50 / 39 / 3$ & $0 / 17 / 44 / 39$ & .0008 \\
\hline$\% \mathrm{DS}$ post-PCI & $16(10,19)$ & $16(8,20)$ & .9 \\
\hline Stent use & $25(92 \%)$ & $36(100 \%)$ & .1 \\
\hline \multicolumn{4}{|l|}{ Electrocardiogram } \\
\hline ST elevation pre-PCI (mm) & $10(7,16)$ & $12(8,18)$ & .2 \\
\hline \%ST post-PCI/pre-PCI & $79(64,98)$ & $19(13,36)$ & $<.0001$ \\
\hline
\end{tabular}

suffered from diabetes mellitus. As a consequence of the study protocol, angiographic criteria of no-reflow (cf myocardial blush grade 0 or 1 ) occurred more frequently in the study group of impaired reperfusion. On the other hand, the use of stents and GP IIb/IIIa receptor antagonists tended to be higher in the patient with good reperfusion. There were no significant differences among the groups with respect to extent of jeopardized myocardium (expressed as the magnitude of summated ST elevation pre-intervention), presence of multivessel disease or cardiac risk factors (besides diabetes), and the angiographic result postintervention.

A total of 11 healthy persons ( 7 men) with a median age of 56 years $(51,60)$ constitutes the control group and had the following cardiac risk profile: $27 \%$ arterial hypertension, 36\% current smoking, 27\% hypercholesterolemia (chol > $220 \mathrm{mg} \%$ ), and no diabetes.

\section{Inflammatory parameters and infarction}

Table 2 summarizes the systemic levels of pro- and anti-inflammatory mediators in control subjects compared to patients with acute myocardial infarction (AMI).

Levels of general markers of inflammation such as leukocyte concentration and CRP were higher in AMI patients than in the control group. Also the IL-10 concentration was slightly higher in AMI patients.

Within the group of AMI patients only, TNF- $\alpha$ differed significantly between patients with versus without reperfusion injury: a median value of 25 versus $13 \mathrm{pg} / \mathrm{mL}$ was observed, respectively.

From a variety of clinical, angiographic, and electrocardiographic baseline factors, logistic regression analysis identified TNF- $\alpha$ levels as the most important independent determinant of the occurrence of reperfusion injury $(P=.001)$. Other independent factors were total ischemic time $(P=.01)$, extent of jeopardized myocardium expressed as the magnitude of summated ST-segment elevation preintervention $(P=.08)$, and treatment with GP IIb/IIIa receptor antagonists $(P=.08)$.

There was no correlation between TNF- $\alpha$ levels and total ischemic time $(P=.8)$ or extent of jeopardized myocardium $(P=.6)$.

There were no reinfarctions and cardiovascular death occurred in two patients. Both patients belonged to the reperfusion injury study group.

\section{DISCUSSION}

Inflammation is increasingly considered to be involved in the pathogenesis of acute coronary syndromes [11]. The present study demonstrates for the first time a strong correlation between proinflammatory status, in particular TNF- $\alpha$ and reperfusion state after recanalization of an occluded infarct artery. A high level of TNF- $\alpha$ was found to be the most important predictor of reperfusion injury in our group of AMI patients, even more 
TABLe 2. Inflammatory status. Data are presented as median value with 25th and 75th percentiles. (a) comparison of control group with AMI patients. (b) inflammatory parameters of AMI patients with impaired reperfusion as compared to AMI patients with good reperfusion.

(a)

\begin{tabular}{lccc}
\hline Variable & Control $(n=11)$ & Infarction $(n=65)$ & $P$ value \\
\hline Leucocytes, x1000/mL & $7.2(6.6,8.2)$ & $9.3(8.2,12.1)$ & .0015 \\
CRP, $\mathrm{mg} / \mathrm{dL}$ & $0.1(0.08,0.15)$ & $0.24(0.09,0.66)$ & .03 \\
TNF- $\alpha, \mathrm{pg} / \mathrm{mL}$ & $17(11,22)$ & $20(11,26)$ & .5 \\
IL-10, pg/mL & $0(0,0)$ & $0(0,18)$ & .01 \\
IL- ra $1, \mathrm{pg} / \mathrm{mL}$ & $1027(702,1359)$ & $1077(668,2290)$ & .5 \\
\hline
\end{tabular}

(b)

\begin{tabular}{lccc}
\hline Variable & Impaired reperfusion $(n=28)$ & Good reperfusion $(n=37)$ & $P$ value \\
\hline Leucocytes, x1000/mL & $9.3(8.1,12.4)$ & $10.0(8.1,12.4)$ & .7 \\
CRP, $\mathrm{mg} / \mathrm{dL}$ & $0.19(0.07,0.7)$ & $0.27(0.1,0.6)$ & .6 \\
TNF- $\alpha, \mathrm{pg} / \mathrm{mL}$ & $25(21,39)$ & $13(7,20)$ & .0001 \\
IL-10, pg/mL & $0(0,12)$ & $0.7(0,21)$ & .4 \\
IL- ral & $1093(840,1713)$ & $978(538,2600)$ & .8 \\
\hline
\end{tabular}

important than previously established determinants such as the extent and duration of ischemia.

Reperfusion injury is characterised by an impairment of microcirculatory flow and by ongoing ischemia and tissue necrosis. Evidence has accumulated that progressive capillary plugging by leukocytes causing capillary noreflow and superoxide radical formation plays a role in this reperfusion injury. Recruitment of neutrophils is regulated through a complex sequence of molecular steps involving the selectins and integrins, which mediate leukocyte rolling and adhesion to the endothelium [6, 12]. There is experimental evidence that cytokines such as IL- 1 and TNF- $\alpha$ are involved in this early stage of neutrophil infiltration by induction of endothelial adhesion molecules [13].

In addition TNF- $\alpha$ may increase the production of reactive oxygen species (ROS) and may so amplify the cascade of local inflammatory response contributing to myocardial reperfusion injury [14]. TNF- $\alpha$ is a proinflammatory cytokine that has been implicated in the pathogenesis of cardiovascular disease, including chronic heart failure, atherosclerosis, sepsis-associated cardiac dysfunction. TNF- $\alpha$ is also produced at the site of ischemia and contributes in a later stage to myocardial dysfunction and cardiomyocyte death in ischemia-reperfusion injury via direct depression of contractility and induction of myocyte apoptosis [15]. One might argue that the observed elevated TNF- $\alpha$ levels in our patients with reperfusion injury are a consequence of higher local production of TNF- $\alpha$ in these patients. However previous work of Neumann et al has shown that local cardiac release of cytokines during reperfusion is too small to affect systemic concentrations in the acute phase of myocardial infarction [16]. In addition no correlation was found between the TNF- $\alpha$ level and the extent or duration of ischemia.
Beyond proinflammatory mediators, we have also measured some anti-inflammatory cytokines that have been implicated in other cardiovascular disease (IL-10 and IL-1ra) [17]. We could not demonstrate any difference among the study groups in our study. This may suggest that those cytokines are not directly involved in the early stage of reperfusion injury. It should however be emphasized that the net effect of any cytokine is dependent on many factors such as the presence of competing or synergistic elements or the timing of release which could not be controlled or measured in the clinical setting of this study.

The results of this study should be considered in the light of a few limitations.

Among potential inflammatory triggers in AMI, this study investigated only a limited number of cytokines (TNF- $\alpha$, IL-10, IL-1ra). Obviously, a number of additional mediators such as cellular adhesion molecules or other cytokines such as the IL-6, IL-8, IL-1 may contribute to the phenomenon of reperfusion injury and remain to be elucidated.

ST-segment resolution was applied to define reperfusion injury. The use of other techniques such as contrast echocardiography or angiography (cf myocardial blush grade) could have changed the distribution of the studied patients. However, previous studies have demonstrated a good correlation between those different diagnostic tools and reanalysis of TNF- $\alpha$ levels using myocardial blush grade as marker of reperfusion injury did not affect the main results [18].

In conclusion, systemic inflammation, in particular high level of TNF- $\alpha$, is strongly associated with the occurrence of reperfusion injury after successful recanalization. Our findings suggest that TNF- $\alpha$ is involved in the triggering and/or amplification of local inflammatory responses 
related to ischemia-reperfusion injury. TNF- $\alpha$ may represent a new target for therapeutic intervention in patients with acute myocardial infarction.

\section{REFERENCES}

[1] Verma S, Fedak PW, Weisel RD, et al. Fundamentals of reperfusion injury for the clinical cardiologist. Circulation. 2002;105(20):2332-2336.

[2] Claeys MJ, Bosmans J, Veenstra L, Jorens P, De Raedt $\mathrm{H}$, Vrints CJ. Determinants and prognostic implications of persistent ST-segment elevation after primary angioplasty for acute myocardial infarction: importance of microvascular reperfusion injury on clinical outcome. Circulation. 1999;99(15):19721977.

[3] Kloner RA, Ganote CE, Jennings RB. The "noreflow" phenomenon after temporary coronary occlusion in the dog. J Clin Invest. 1974;54(6):14961508.

[4] Ambrosio G, Tritto I. Reperfusion injury: experimental evidence and clinical implications. Am Heart J. 1999;138(2 pt 2):S69-S75.

[5] Kloner RA, Giacomelli F, Alker KJ, Hale SL, Matthews R, Bellows S. Influx of neutrophils into the walls of large epicardial coronary arteries in response to ischemia/reperfusion. Circulation. 1991;84(4):1758-1772.

[6] Vermeiren GL, Claeys MJ, Van Bockstaele D, et al. Reperfusion injury after focal myocardial ischaemia: polymorphonuclear leukocyte activation and its clinical implications. Resuscitation. 2000;45(1):3561.

[7] van 't Hof AW, Liem A, de Boer MJ, Zijlstra F. Clinical value of 12-lead electrocardiogram after successful reperfusion therapy for acute myocardial infarction. Lancet. 1997;350(9078):615-619.

[8] Feldman LJ, Coste P, Furber A, et al. Incomplete resolution of ST-segment elevation is a marker of transient microcirculatory dysfunction after stenting for acute myocardial infarction. Circulation. 2003;107(21):2684-2689.

[9] Claeys MJ, Vrints CJ, Bosmans JM, Conraads VM, Snoeck JP. Aminophylline inhibits adaptation to ischaemia during angioplasty. Role of adenosine in ischaemic preconditioning. Eur Heart J. 1996;17(4):539-544.

[10] van 't Hof AW, Liem A, Suryapranata H, Hoorntje JC, de Boer MJ, Zijlstra F. Angiographic assessment of myocardial reperfusion in patients treated with primary angioplasty for acute myocardial infarction: myocardial blush grade. Circulation. 1998;97(23):2302-2306.

[11] Pearson TA, Mensah GA, Alexander RW, et al. Markers of inflammation and cardiovascular disease: application to clinical and public health practice: A statement for healthcare professionals from the Centers for Disease Control and Prevention and the American Heart Association. Circulation. 2003;107(3):499-511.

[12] Frangogiannis NG, Smith CW, Entman ML. The inflammatory response in myocardial infarction. Cardiovasc Res. 2002;53(1):31-47.

[13] Dinarello CA. Proinflammatory cytokines. Chest. 2000;118(2):503-508.

[14] Nian M, Lee P, Khaper N, Liu P. Inflammatory cytokines and postmyocardial infarction remodeling. Circ Res. 2004;94(12):1543-1553.

[15] Herrera-Garza EH, Stetson SJ, Cubillos-Garzon A, Vooletich MT, Farmer JA, Torre-Amione G. Tumor necrosis factor-alpha: a mediator of disease progression in the failing human heart. Chest. 1999;115(4):1170-1174.

[16] Neumann FJ, Ott I, Gawaz M, et al. Cardiac release of cytokines and inflammatory responses in acute myocardial infarction. Circulation. 1995;92(4):748-755.

[17] Opal SM, DePalo VA. Anti-inflammatory cytokines. Chest. 2000;117(4):1162-1172.

[18] Angeja BG, Gunda M, Murphy SA, et al. TIMI myocardial perfusion grade and ST segment resolution: association with infarct size as assessed by single photon emission computed tomography imaging. Circulation. 2002;105(3):282-285. 


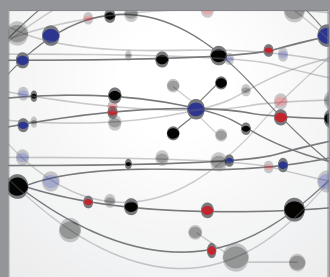

The Scientific World Journal
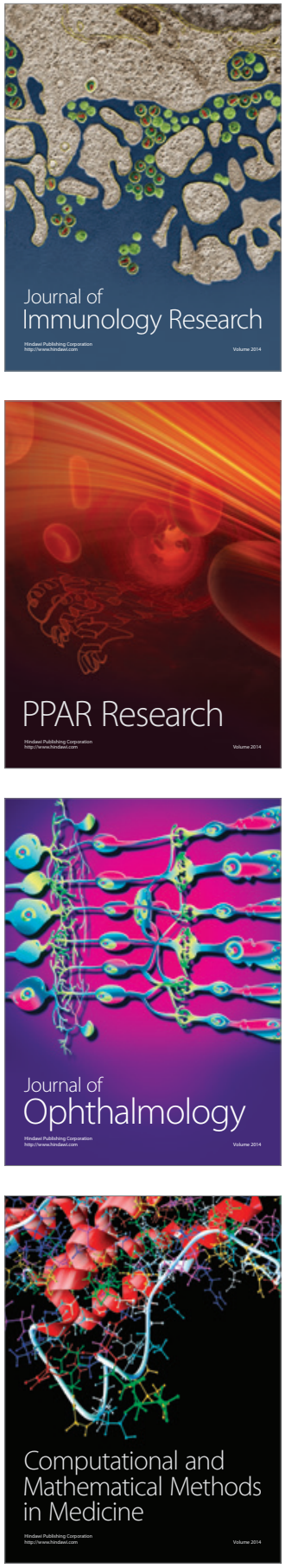

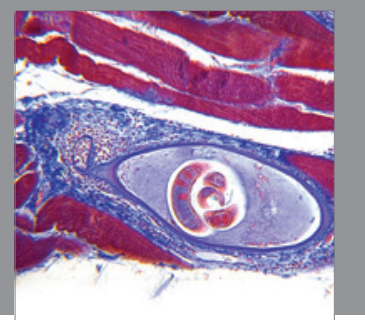

Gastroenterology

Research and Practice
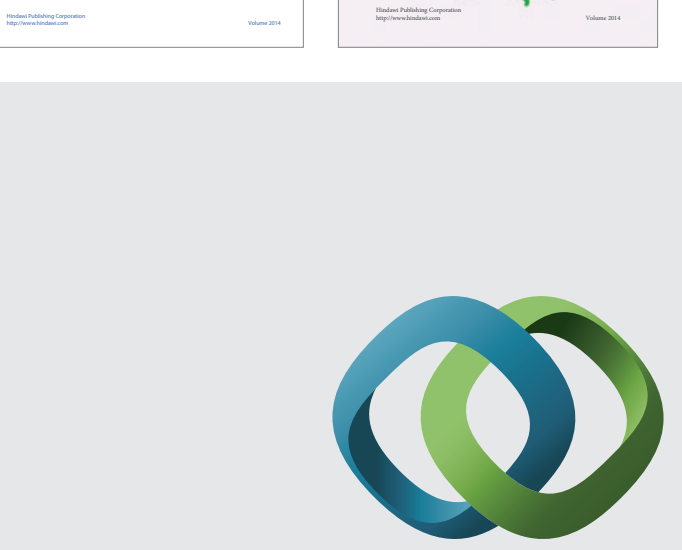

\section{Hindawi}

Submit your manuscripts at

http://www.hindawi.com
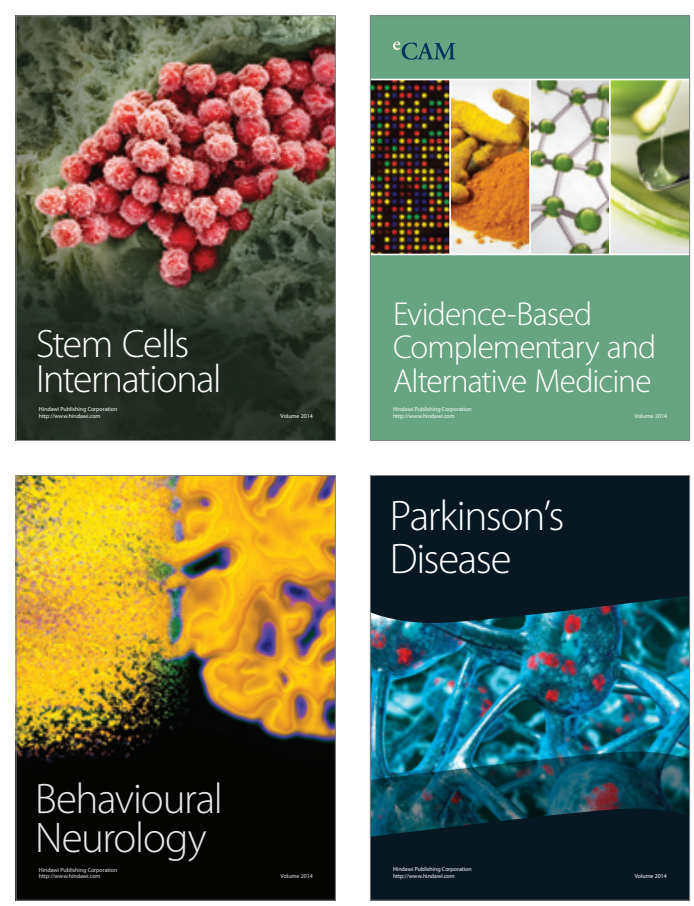

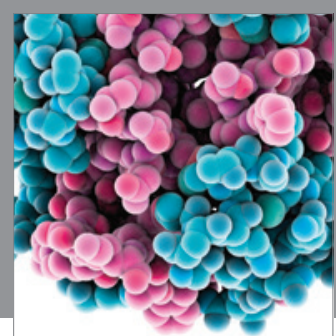

Journal of
Diabetes Research

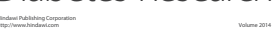

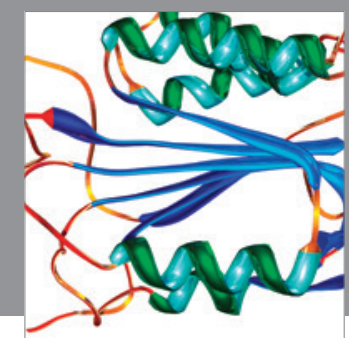

Disease Markers
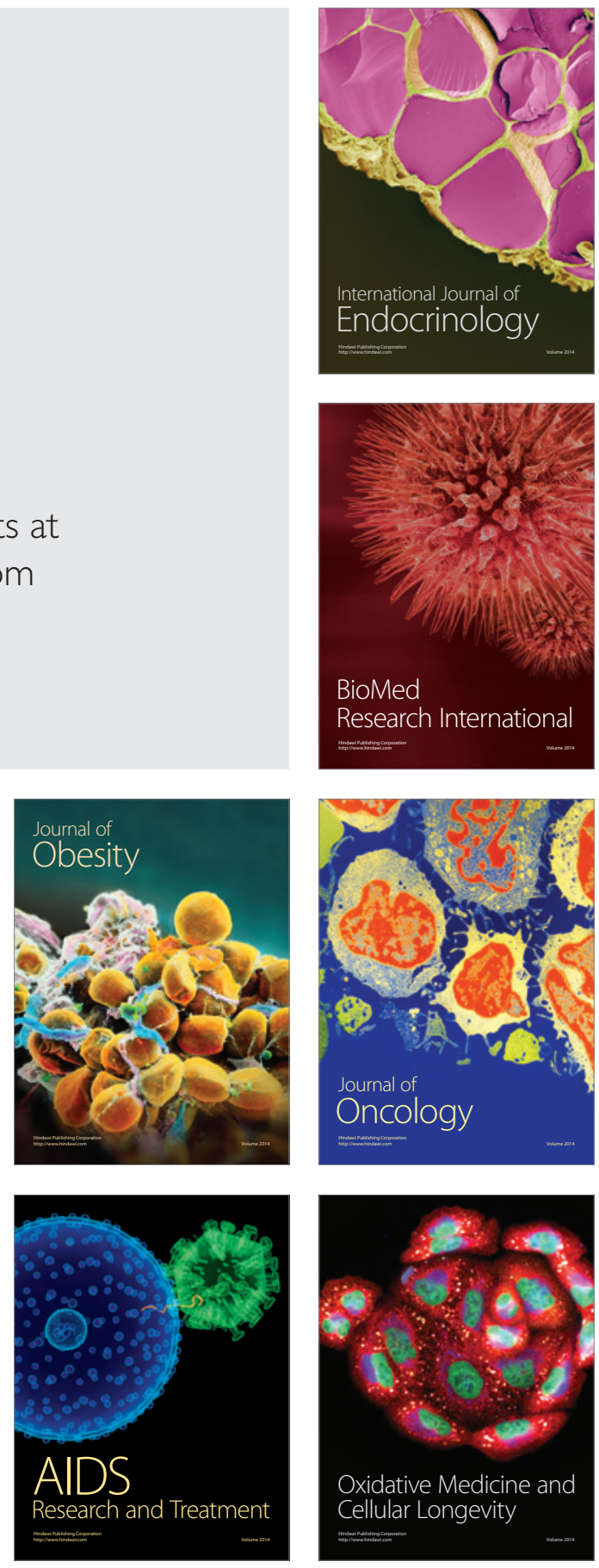